Preprint. Final version appears as:

Bull, P.A., Morgan, R.M., Sagovsky, A. \& Hughes, G.J.A. (2006) The Transfer and Persistence of

Trace Particulates: Experimental studies using clothing fabrics. Science and Justice, Vol 46: 185-195

\title{
The Transfer and Persistence of Trace Particulates: Experimental studies using clothing fabrics.
}

\author{
Bull, P. A.*, Morgan, R. M., Sagovsky, A. and Hughes, G. J. A. \\ Oxford University Centre for the Environment, Dyson Perrins Building, South Parks
}

Road, Oxford, OX1 3QY

* Author for correspondence

$\underline{\text { Peter.bull@hertford.ox.ac.uk }}$

Tel: +44 1865275840 


\title{
The Transfer and Persistence of Trace Particulates: Experimental studies using clothing fabrics.
}

\author{
Bull, P. A.*, Morgan, R. M., Sagovsky, A. and Hughes, G. J. A. \\ Oxford University Centre for the Environment, Dyson Perrins Building, South Parks \\ Road, Oxford, OX1 3QY
}

\begin{abstract}
A series of experiments were conducted using various types of pollen grain, powder and metal particulates to determine their persistence on a range of different types of materials. The decay curves generated in the repeated experiments all broadly conform to those obtained and already presented in the literature for fibres and glass particulates. The experiments presented in this paper were conducted for up to 647 hours for individual runs and it was found that a general rule existed that it was the material type rather than the particulate type that was the major controlling factor in the persistence of particles. For the pollen grain experiments the overall theory is sustained; there is a significant loss initially followed by a slower decay although this is not precise given the varied nature of palynomorph types. The manner by which the particulates were introduced onto the material had an initial immediate effect, however, the persistence of these particulates over longer periods of time was generally unaffected.
\end{abstract}

Keywords: Forensic Science; transfer; persistence; sediment; pollen; lighter flint particles 


\section{Rationale}

The advent of new and ever more sophisticated analytical machinery has increased the potential power of forensic geoscience analysis. Comparatively little has changed in the microscopic investigation of the presence and persistence of particulates on various articles of clothing, footwear or motor vehicles. The work of Pounds and Smalldon [1 - 3], Robertson and Grieve [4], Robertson and Roux [5], and Houck [6] summarise concisely the commonly accepted basis for an understanding of the factors which influence the transfer and persistence of fibres. Other studies have investigated the presence, persistence and transfer of other particle types on various fabrics and articles of clothing and these seem to provide comparable results to those obtained in fibre experiments (for example, for glass particulates, see; [8], [9], [10], [11], [12, [13], [14], [15] [16]: for paint particulates, see; [17]; for foam particulates, see: [18]; and for hair, see: [19]). However, there are little data to support the view that there is no need to change our present understanding of particulate decay rates from the default situation where various different geoscience trace particulates act in the same way as recorded for fibres or glass particulates.

The inference presented in these published papers (referring to glass, paint, foam, hair etc.) is that in the first instance, particulates are shed from clothing rapidly (minutes) and then subsequently shed more slowly, with varying percentages of particulates remaining after several hours. The overall effect of the decay curve appears to be one of exponential decrease. There are however, a number of factors which have been reported to affect the persistence and decay curves of small particulates and these are well summarised by Houck [6]. He considers that the size and texture of the material being transferred may affect the persistence of such trace materials and links this to the type of surface on which the material is being retained. Further, he considers whether the particulates can be easily removed (and whether a suspect has tried to remove them). Crucially, he identifies the importance of the length of time interval between the offence and recovery of the retained material and links this to the degree of activity undertaken by the suspect or the victim following particulate transfer.

The experiments presented in this paper are in response to a series of specific casework requirements in which it was deemed necessary to determine the relevant transfer and persistence of small sedimentary particles (soils, natural clays, silts and 
sands, pollen and metal particulates), from a number of different types of clothing. Specifically, this paper seeks to identify not only the transfer, persistence and relevant abundance of particulates on clothing over time, but also whether a general rule can be introduced for all such trace particles of generally similar size (less than $100 \mu \mathrm{m}$ diameter) and occurrence (glass, paint, fibres, hair, soils, clays, silts, sands, pollen and other anthropogenic materials). There is a need to consider whether geoscientific trace materials act in the same, or different, manner as those anthropogenic particulates already documented in the literature. We need to investigate the contention that geoscientific particulates are, by default, acting in the same way as these other materials.

The experiments are provided in the order that they were undertaken. No anomalies have been smoothed in order to stress the importance of the development of an accurate experimental design. Each experiment builds upon the lessons learned from the previous case and account is taken of the different types of particulate and recipient materials, the different lengths of duration of persistence (and this necessitates different observation time increments), and the affects of one-way transfer mechanisms.

\section{The persistence of pollen grains on various types of material}

\subsection{Experiment 1: The persistence of pollen grains of daffodil (Narcissus Sp) and}

\section{Dutch tulip (Tulipa)}

The transfer and preservation of both Dutch tulip and daffodil pollen was studied using clean (tested) swatches of six different material types. Each flower type was oven dried for 48 hours to separate the pollen grains and provide a standard moisture content. The pollen was brushed onto each material swatch which itself was attached to a coat. For this experiment acrylic, cotton, denim, nylon, polyester and wool were used. The acrylic swatch was $93 \%$ acrylic, $6 \%$ nylon and $1 \%$ elastane with a medium texture, open weave from a knitted woollen jumper garment; the cotton was $100 \%$ cotton with smooth/medium weave from a standard t-shirt garment; the denim swatch was $100 \%$ cotton with coarse open weave from denim trousers; the nylon swatch 
consisted of $94 \%$ nylon, $6 \%$ lycra/elastane in an open weave garment which were ladies tights; the polyester was $100 \%$ polyester, rough textured, open weave knitted jumper garment; the wool swatch was $100 \%$ wool, rough textured, knitted jumper garment.

The coat was worn both in and outdoors for a total of seven days. No overcoat was used. Adhesive tape strips mounted on SEM stubs were used to collect the pollen after designated periods of time from a pre-marked specific location on the swatch. Each taping was then cut into $0.5 \mathrm{~cm}^{2}$ and attached to an electron microscope stub for viewing. The 48 samples ( 24 of each pollen type) were then viewed by scanning electron microscopy at $\mathrm{x} 70$, effecting a $500 \mu \mathrm{m}^{2}$ viewing area. Raw counts and computed percentages are presented in Tables 1 and 2.

All six materials show a general decrease in pollen numbers counted for each of the 24, 96 and 168 hour observations, although acrylic, wool and cotton retain surprisingly high percentages of both tulip and daffodil pollen after the equivalent of seven days of continuous wear (168 hours). Table 3 summarises the average percentage loss for both tulip and daffodil pollen grain types for all six material types used in these experiments. These results are indeed very encouraging for potential use in forensic casework. However, to derive full pollen spectra from clothing after a lapse of seven days could potentially be very difficult and indeed, possibly misleading. Account must be taken of the relevant abundances of different types of pollen (wind or insect dispersed), although individual events (such as brushing against a particular flowering plant or shrub) may well be recorded accurately and be of great significance for forensic reconstruction.

What is established in this experiment however, is the fact that pollen does persist on all of the material types used for a period of time in excess of 168 hours. A more detailed sampling time interval is needed to determine the nature of pollen grain decay over time. Furthermore, table 3, which shows the average percentage retention for daffodil and tulip for six material types somewhat disguises the actual variance of the percentage of pollen retained on the different materials for each of the two pollen types tested. Thus the average percentage retention after 168 hours of 
experimentation for tulip of $7.37 \%$ and daffodil of $10.83 \%$ (table 3 ) belies the fact that the variance of pollen retention for tulip and daffodil (standard deviation of 6.20 and 9.54 respectively) for each of the six materials is of a magnitude as to suggest that it is perhaps the material and not the pollen type that is the predominant factor in particle retention. This suggestion is explored more fully in experiment 2 .

\subsection{Experiment 2: The persistence of pollen grains on various materials}

This series of experiments involved the investigation of the persistence of four pollen grain types (lily, sambucus, pine and hydrangea) on five different materials (the same broad types of material used in experiment 1: cotton, acrylic, wool, nylon tights and polyester. In this experiment denim was not used. For more detail of these experiments see [7]). This experiment was conducted over a longer period of time and sampled at smaller time periods than that employed in experiment 1. Once again small swatches $(22 \times 13 \mathrm{~cm})$ of each material were used and pinned on outer clothing and worn during the day. The four pollen types were chosen to incorporate a range of pollen grain sizes and also because they provided sufficient pollen for use in the experiments. Hydrangea (Hydrangea carpentaria californica (Ladhams variety)) pollen has a diameter of approximately $10 \mu \mathrm{m}$; Sambucus pollen (Sambucus nigra),

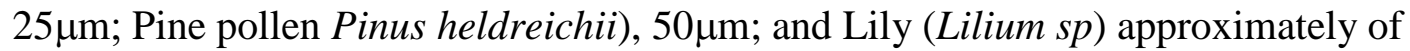
$100 \mu \mathrm{m}$. All had a general oval form.

The four pollen types were dried in an oven for 48 hours and the dried pollen was sieved onto the material swatches (to produce an even distribution of pollen). A small brush was used to lightly brush the pollen horizontally and vertically across the patch to simulate a person brushing against the flower, rather than simulating a case of pollen falling onto the material (as was the case in experiment 1). After designated time periods adhesive taped SEM stubs were used to collect particulates in predesignated columns on the material swatch.

Figure 1 provides the decay curves of each material type for all pollen experiments conducted and also the decay curve of each pollen type for all materials tested. It is immediately apparent that the variance from the decay curve trend is larger for the 
material types (Figure 1f-1i) than the variance for the pollen types using specific materials (Figure 1a-1e). It is clear then, that the dominant factor controlling the decay rate of pollen grains on clothing is the type of material from which the clothing is made. Wool (Figure 1i) has the least variance in its decay trend irrespective of pollen type, although there is remarkably little variance in the decay trends for cotton, acrylic, nylon and polyester. It is of interest to note that for all pollen types and material types, pollen grains were recovered even after 647 hours of wear.

Table 4 provides summary data of the average percentage retention for the four pollen types used in this experiment aggregated from the results obtained from the experimental runs of each of the five material types. It can be seen that the average decay of pollen is rapid in the initial outset but decay is of a more modulated linear nature after the first day. The apparent increase in the percentage of pollen retained after certain time periods, evident from table 4, may be due to the shedding of pollen from one area of the material and its deposition onto another area of the material. It is easy to forget that particulates that are shed from an area of clothing may well reattach themselves to another area of clothing (such as from a blouse to a skirt, or from a shirt to trousers) rather than be lost completely. Although all experiments conducted in this phase of investigation were conducted indoors in laboratories of controlled humidity and temperature surroundings, it is quite possible that drier weather conditions may affect pollen grains such that they are stuck more readily to the adhesive tape. Certainly the rate of collection of particulate traces on tape may be reduced if the tape or garment is damp. Formal investigation of this assertion would prove interesting in further experimentation.

Experiments were conducted using the same pollen types with four of the material swatches (cotton, acrylic, wool and polyester) whereby the materials were washed following deposition of the pollen onto the material swatch. The materials subjected to dry-cleaning, machine washing and hand washing and each of these three washing procedures were undertaken prior to taping of the swatches for the presence of pollen grains. Tables 5 and 6 show that some pollen grains do remain on all types of material even after washing, although the number of grains recovered are low (these results are in concord with the observations made by others (Wiltshire pers comm.)). There was no testing of the washing machine prior to experimentation, and this 
seemed to vindicated by the lack of different palynomorphs recovered (other than those used in the experiments) after the washing procedure. It would be important in case work to assess such prior event contamination but by the very nature of the postevent reconstruction by crime scientists it is not possible in a real case scenario to have blank tests available prior to the event unless the machine were brand new (which has happened in the authors' experience on one occasion).

\section{Particles of anthropogenic source}

Two experiments were undertaken to determine the persistence of fine-grained particulates on different types of material. The experiments were conducted in response to a case under investigation. In the first experiment, standard scene of crime fluorescent powder (manufactured by SCE Ltd) with a mean grain size $14 \mu \mathrm{m}$ (with a particle diameter range between 5 and $25 \mu \mathrm{m}$ ) was used as a proxy for fine silts. The advantage of using fluorescent powder was that it could be observed and quantified under conditions of ultra-violet light. The second experiment sought to investigate the decay of lighter flint particles, particularly of cerium and lanthanum, which were discharged by the action of striking the flint of a disposable lighter.

Various laboratory tests were undertaken prior to the experimental runs to determine the affect of the powder upon various soil types, particle sizes, different types of material swatch and shoe sole compositions. Further, the powder was subjected to different moisture levels. No discernable difference was identified with any variant of material type, moisture level or grain size with regard to persistence, spreading capability, tenacity, transfer or detection during experimentation.

\subsection{Experiment 3 Fluorescent powder persistence}

Much care was taken to develop a procedure by which fluorescent powder could be introduced onto different material swatches. It was found that by mixing the powder with domestic quality ground flour that an even distribution of particulates could be 
achieved across a swatch of material. For this experiment, nylon, wool, PVC and a viscose/nylon/lycra mix material swatches were used (see figure 2). No obvious chemically induced attractive properties between particulates and garments were recognised prior to experimentation.

The powder mixture was sprinkled onto a sheet of paper using muslin butter cloth as a sieve (this was found by repeated experimentation to be the most even method of powder introduction onto materials). After a control photograph was taken of each swatch using a digital camera, the various fabric swatches were placed on top of the powder and a person then sat on the fabric for one minute (the swatches were then pinned to a laboratory coat and worn). Photographs were taken of the swatches under UV light after each hour of wear for 24 hours. The photographs were pixelated using Corel Photo-Paint 9 and the number of particles in each image were computed (as a function of pixel brightness) and are provided as both raw and percentage data. Tables 7 and 8 present the results of the persistence of fluorescent powder, achieved in these experiments and shows that even after 24 hours there was still $12 \%$ retention on PVC, $39.7 \%$ on wool, $12.4 \%$ on the viscose/nylon/lycra mix and $12.7 \%$ on nylon. Each value in tables 7 and 8 is a mean of five individual experimental runs.

The decay curves derived for each of the four materials approximates the same shape decay curve (Figure 3 ) as presented for experiment 2 in this paper and for previously published decay curves for fibres and glass. There is however, a variation in the amount of material that remains after 24 hours dependent on the type of recipient material used in each experiment and, as inferred from the interpretation of the results in previous experiments, the controlling factor appears to be the type of material used in each experiment, rather than the particulates used.

\subsection{Experiment 4 Persistence of lighter flint particles}

For this experiment, three swatches $(15 \times 10 \mathrm{~cm})$ of a viscose material and of a nylon material were used in order to further investigate the trends suggested in experiment 3. Relevant control tapings of the swatches were also undertaken, and the swatches were then pinned onto a laboratory coat after lighter flint particles were flicked onto 
the material from a 'Clipper' lighter. A large amount of debris is produced by each flick of the lighter, but for this experiment only the rounded cerium and lanthanum particles were counted. These particles range in size from only $1-2 \mu \mathrm{m}$ in diameter to $40-50 \mu \mathrm{m}$ in diameter. The material was taped vertically in pre-drawn columns after designated time periods of wear. The results are presented in table 9, and each value represents the mean of three different runs for each experiment. The agglomerated means of cerium and lanthanum particle persistence (table 10) for each of viscose and nylon show that between $20 \%$ and $30 \%$ of the lighter flint particulates still remain after 18 hours of wear. Once again, the decay curve (figure 4) shape approximates the same as for the other three experiments presented in this paper using fluorescent powder and seven different types of pollen and accords with the findings of previously published work on fibres and glass. The main difference between the cerium and lanthanum particles and the pollen, fluorescent powder, fibres and glass particles is that the lighter flint particles have specific gravities in excess of 6.0, whilst the others have specific gravities of less than 2.7. It would appear that the specific gravity of such small particles certainly does not mitigate against persistence of these particulates, but rather may well enhance the preservation of the heavier particles within the fibres of the material swatches.

In order to determine whether the cerium/lanthanum ejecta were adhering to the fabric as a result of their residual heat melting the fabric, ejecta were fired from the lighter onto clingfilm plastic stretched over a beaker. Microscopic inspection showed that there was no melting of the very thin film. It was judged that melting onto fabric would also be unlikely. No melting phenomena were observed in any of the experimental runs.

\section{Discussion}

The four experiments outlined above have, to a certain extent, evolved one upon another in response to the results and inferences obtained in each experiment. The initial question outlined at the beginning of this paper concerned whether it would be possible to look at small (less than $100 \mu \mathrm{m}$ ) trace particulates and investigate the decay rates of these particles using different types of material and to determine whether 
there may be a general rule of particle decay behaviour. It is apparent that finegrained particulates (six different types of pollen, fluorescent silt-sized powder and lighter flint debris) remain on clothing for a surprisingly long time period even when the clothing is worn. The decay curves identified for each type of material conform to the general shape of those already identified in the literature for fibres, hair and glass. Some of these present experiments however, were repeated over hundreds of hours and it appears that the type of material has a far greater influence upon the relative decay curves through time than does the type of particulate. In order to identify whether these relationships are meaningful we have assessed the strength and type of relationship between particle persistence and time to determine whether the 'weakly bound' and 'strongly bound' model provided by Pounds and Smalldon [3] concerning fibres can be identified.

\subsection{Statistical evaluation of the decay curves for both fluorescent powder and $\underline{\text { lighter flint particles }}$}

When statistical tests were applied to the decay curve data for lighter flint (cerium and lanthanum) particles, they showed a remarkably high degree of correlation between the hours of wear and the percentage of lighter flint particulate retention. Spearman's Rank Correlation Co-efficient provides a correlation figure of $97 \%$ for the viscose material and 94\% for the nylon material (both statistically significant at the 99\% level using a 1-tail t-test).

The smoothness of the decay curve (figure 4) also allowed the formulation of an equation. It is clear that there is not a simple formula for the decay of these particles. The curve for the first 6 hours (see figure 4) appears to be exponential in the form:

$$
\mathrm{n}=\mathrm{n}_{0} \mathrm{e}^{-\lambda \mathrm{t}}
$$

Where $\mathrm{n}=$ the percentage of particles remaining, $\mathrm{n}_{0}=$ the original percentage of particles (in this case always 100 ), $\mathrm{e}=$ exponential, $\lambda=$ the decay variable (equal to the gradient of the graph of $\mathrm{x}$ against the natural $\log$ of $y$ ) and $t=$ time. 
However, the second half of the curve (where $t$ is greater than or equal to 6), is a straight line in the form:

$$
\mathrm{y}=\mathrm{mt}+\mathrm{c}
$$

where $\mathrm{m}=$ the gradient, $\mathrm{t}=$ time and $\mathrm{c}$ is constant.

It is possible to test whether the graph showing the decay of lighter flint on both the viscose and nylon material (figure 4) is showing an exponential decay for the first 6 hours, by computing a graph of the natural log of the y value (in this case the percentage of particulates remaining). If the resultant graph is a straight line then the original decay is exponential [20]. The results of such a procedure are that the graphs for both the viscose and nylon material are indeed a straight line (the line equations are respectively $\mathrm{y}=-0.19 \mathrm{x}+4.63$ and $\mathrm{y}=-0.16 \mathrm{x}+4.60)$.

Thus both decay curves will have an equation of the form:

$$
\mathrm{n}=\mathrm{n}_{0} \mathrm{e}^{-\lambda \mathrm{t}}
$$

The value of $\lambda$, the decay variable, can be determined from the gradient of the natural $\log$ graph which is equal to the coefficient of $\mathrm{x}$ in the equations provided above, and are in this case 0.19 and 0.16 . Thus the formula for the first six hours of decay:

$$
\begin{array}{ll}
\text { For viscose material } & \mathrm{n}=100 \mathrm{e}^{-0.19 t} \\
\text { For nylon material } & \mathrm{n}=100 \mathrm{e}^{-0.16 t}
\end{array}
$$

The co-efficient of determination $\left(\mathrm{r}^{2}\right)$ for viscose material is $98.56 \%$ and for nylon material is $98.96 \%$ indicating that the variation in the natural log of the percentage of particles remaining for the viscose and nylon materials is determined by the variation in the length of time that those particles have been present on the fabric. This is significant at the $99.8 \%$ confidence level (computed by t-test). The decay curve of particles is therefore exponential over the first six hours.

The second part of the decay curve (where $t$ is greater than 6) is a straight line of the form:

$$
\mathrm{y}=\mathrm{mt}+\mathrm{c}
$$


Thus the linear decay of the particles after six hours on the viscose material as shown in figure 4 approximates a line $y=-0.79 x+39.70$ and the linear decay of the particles after six hours on the nylon material approximates to $y=-0.83 x+46.11$

The computed co-efficients of determination for this linear decay is 98.23 (viscose) and 99.39 (nylon), both of which are shown to be significant to $98 \%$ confidence level.

These can be amalgamated into a statement thus:

For the viscose fabric, if $\mathrm{t}$ less than or equal to 6 , then $\mathrm{n}=100 \mathrm{e}^{-0.19 \mathrm{t}}$ but if $\mathrm{t}$ is greater than 6 then $\mathrm{y}=-0.97 \mathrm{t}+39.70$ :

For the nylon fabric, if $\mathrm{t}$ less than or equal to 6 , then $\mathrm{n}=100 \mathrm{e}^{-0.16 \mathrm{t}}$ but if $\mathrm{t}$ is greater than 6 then $y=-0.83 t+46.11$

The multiple stage of the decay of particles found here is supported by the findings of Pounds and Smalldon [3] although they suggest a three-stage decay for fibres (weakly bound, bound and strongly bound). They found that the time taken for the fibres to be lost corresponded to the strength of the bond between fibre and clothing. Therefore, the shape that is adopted by any curve of decay will depend upon the proportion of fibres which are initially present in each of the three states [3]. Although Pounds and Smalldon [3] remark that in the 'weakly bound' state the fibres decayed 'so rapidly it was difficult to study' ([3] p204), this state does not seem to exist for the lighter flint particulates studied in these experiments where the initial decay curve is exponential. Interestingly these findings are supported by the work of Dachs et al. ([19] p34) who show that the loss of hairs in experiments 'follows an exponential decay curve on non-wool garments' they do not however examine the decay curve after the first five hours of wear to confirm our findings on the decay of lighter flints of a two-stage process.

The two different decay curves may indicate two different decay processes occurring either in tandem or in succession. It would be reasonable to suppose that the exponential decay at the start of the experiments is caused by particles being shed which are sitting on the surface of the fabric and decaying in an exponential manner. It may suggest that the slower linear decay in the second half of the experiment could be the result of particles that have become lodged in the weave and hence decay at a different rate. In the period from six hours onwards, our experiments show that the 
rate of decay of flint particles is governed by the characteristics of the fabric, perhaps mirroring the behaviour of the decay of pollen particles outlined above.

\subsection{Statistical evaluation of decay curves for pollen grains on different material types}

Figure 1 provides numerous decay relationships for both material type and pollen decay over time and it can be seen that the decay curve approximates to what would be expected from curves provided in the literature for fibres and glass. Since these experiments were conducted over a much longer period of time than those that have been published to date, we sought to determine whether the decay curves could approximate an initial exponential decay followed by a more linear pattern as identified for the lighter flint experiments above. It is also important to note that in these pollen decay experiments, the pollen grains were only lightly brushed onto the surface of the material (approximating the 'weakly-bound' and 'bound' stages identified by Pounds and Smalldon [3]). Graphical and statistical analyses were conducted upon the pollen persistence data (in a similar manner to that undertaken for lighter flint particles). It was clear that the exponential decay curve for the first six hours was not present for any pollen or material type since the curves were not 'smooth'; nor was there a fitted linear relationship for second stage of decay as found in the lighter flint experiments. We judge that since the pollen grains were only lightly brushed onto the surface of the material that they were shed relatively rapidly in the initial hour or two (as mentioned by Pounds and Smalldon [3]) but nevertheless did remain present on the materials for a considerable amount of time. Further, the non-linear relationship of the second stage of the decay curve may well be explained by the re-incorporation of pollen grains which have fallen from one area of the material to another. Pollen grains may not just decay from the closed system of the material, but may re-incorporate themselves into the material in large numbers if they were initially introduced by weak bonding mechanisms. Pollen grains will fall from one type of clothing to for example, skirt or trousers, and given the various activities that can occur after the initial introduction of the particulates onto the material they can easily be incorporated into the more strongly-bound associations identified by Pounds and Smalldon [3] and shown conclusively in these repeated experiments. 
Care must be given however, to the possibility that the collection of particulates onto adhesive tape may be effected by fluctuations in humidity and temperature which occur most continuously in the natural environment and hence may affect forensic interpretation of data. Further experimentation is needed to test the assertion that the pollen grains fall from some clothing to be reincorporated at a lower level, perhaps on trousers, skirts or lower garments.

\section{Conclusions}

The main aim of this paper was to identify whether a general rule of particulate decay could be advanced using a range of different small particles and a variety of different types of recipient material. A template existed in the published literature for decay curves of glass, fibres, hair, foam and paint particulates, and these all approximated the same exponential curve of decay of particles over time. Here, we extended the length of time over which particle decay could be monitored and found that for various pollen types up to 647 hours of monitoring resulted in the identification of the persistence of pollen grain on different materials.

It is apparent that the type of material rather than the type of particulate is the major controlling influence in particulate preservation. Further, pollen grains (and perhaps also by inference other particles) do survive different forms of washing. Similar longer term decay curves could be identified for fine, silt-sized fluorescent particles and for lighter flint debris.

The results of the experiments presented in this paper, conform with a two-stage model outlined by Pounds and Smalldon [3] and provide relevance for forensic investigation of trace particulates which are sampled from clothing many hours or even days after a specific event rather than only a three or four hour period as presently presented in the literature. Once attached, particles persist on most types of material for considerably longer than has been previously considered. A controlling factor in this persistence is shown also to be related in part to the method of transfer of particulates onto material is effected. The longevity of particle persistence presented in this paper adds considerable potential to the relevance of trace particle 
analysis. However, the specific data points identified in the decay curves for different particulate types and for different garments were aggregated means and as such variation occurred between experimental runs at each given time and for each different scenario. These data should not be used as predictive and absolute points of reconstruction, they indicate trends.

A further consideration is also needed: The very persistence and tenacity of small trace particles (whether they be anthropogenic in origin such as glass, fibres, hair, paint etc. or whether they be naturally occurring soil, sediment or pollen) will inevitably mean that without the cleaning of their host materials or garments that multi-provenance contamination or palimpsest reflections could be present in forensic case analysis. Larger amounts of material present on host garments may well imply a more recent provenance origin, equally, a very small amount of material present most certainly could involve pre-forensic event additions. The specific characteristics of the particulates may themselves differentiate various provenances of that material, so long as the analytical techniques of analysis do not require the gross homogenisation by solution or grinding rendering any analysis meaningless. Careful and selected analysis however utilising non-homogenisation techniques may indicate small percentage (and perhaps long time) particulate additions. This further complicates any Bayesian approach to data interpretation. The significance of the presence (or indeed absence) of geoforensic particulates on host garments or clothing must be established in the light of these findings before any assessment of the likelihood ratio, or Bayesian interpretation of the evidence can be made. As ever, each case is individual, the real-life scenario is not absolute and prediction of the transfer, persistence, recovery and accurate interpretation of data derived from forensic analysis requires careful consideration rather than uniform statistical application.

\section{Acknowledgements}

The authors would like to acknowledge discussion and comments with Patricia Wiltshire, Adrian Parker, Chris Jackson and Matt Morgan. RM acknowledges financial support from the Bruce, Julia and Mortimer May Senior Scholarship at Hertford College Oxford. We acknowledge the help and suggestions of the staff of 
the Botanical Gardens, University of Oxford. We acknowledge the very helpful comments of the two anonymous reviewers of this paper.

\section{List of Figures and Tables}

Figure 1 The decay curve of pollen grains on different fabrics over a period of 647 hours (4 weeks) (Compiled from Candidate 2004).

Figure 2 SEM images of four different fabric types; A. Nylon; B. Wool; C. PVC and D. Nylon/Viscose.

Figure 3 The decay curve for fluorescent powder particulate retention over a period of 24 hours on four different types of material. (diamond $=$ nylon, square $=$ PVC, triangle $=$ wool and cross $=$ viscose $/$ nylon/lycra $)$

Figure 4 The decay curve for lighter flint particulate retention over a period of 18 hours on viscose and nylon material (diamond $=$ viscose material and square $=$ nylon material).

Table 1 The persistence of Dutch Tulip pollen (Tulipa).

Table 2 The persistence of Daffodil pollen (Narcissus).

Table 3 Average $\%$ retention for both daffodil and tulip pollen grains with all four material types.

Table 4 Average percentage retention for the four pollen grain types for 5 different material types used.

Table 5 Persistence of pollen grains: Aggregate after three runs (all materials).

Table 6 Total amount of pollen grains recovered from different materials after three runs of washing.

Table 7 Persistence of fluorescent powder on four different materials over a 24 hour period (each value is a mean of five experimental runs).

Table 8 Percentage persistence of fluorescent powder on four different materials over a 24 hour period (each value is a mean of 5 experimental runs).

Table 9 Lighter flint particle persistence (in numbers) on nylon and viscose material for an 18 hour period (each value is the mean of three different runs).

Table 10 Lighter flint particle persistence (mean percentage recovered, each from three experiments) on nylon and viscose material for an 18 hour period. 


\section{References}

[1] Pounds, C. A. and Smalldon, K. W. The transfer of fibres between clothing materials during simulated contacts and their persistence during wear, part I - Fibre transference. Journal of the Forensic Science Society 1975a; 15: 17-27.

[2] Pounds, C. A. and Smalldon, K. W. The transfer of fibres between clothing materials during simulated contacts and their persistence during wear, part II. Journal of the Forensic Science Society 1975b; 15: 29-37

[3] Pounds, C. A. and Smalldon, K. W. The transfer of fibres between clothing materials during simulated contacts and their persistence during wear, part III $-\mathrm{a}$ preliminary investigation of the mechanisms involved. Journal of the Forensic Science Society 1975c; 15: 197-207

[4] Robertson, J. and Grieve, M. C. The Forensic Examination of Fibres. London: Taylor and Francis, 1999.

[5] Robertson, J. and Roux, C. Transfer and Persistence. In Siegel, J. A., Saukko, P. J. and Knupfer, G. C. (eds) Encyclopedia of Forensic Sciences. Academic Press, 2000 pp834-838

[6] Houck, M. Mute Witness: Trace Evidence Analysis. London: Academic Press, 2001.

[7] Candidate no. 22000 Forensic Palynology: the persistence of pollen.

Unpublished dissertation, School of Geography Library, University of Oxford, 2004.

[8] Koons, R. D., Buscaglia, J., Bottrell, M. and Miller, E. T Forensic glass

comparisons. In Saferstein, R (ed) Forensic Science Handbook. $2^{\text {nd }}$ edition

Englewood Cliffs: Regents/Prentice Hall, 2002.

[9] Curran, J. M., Hicks, T. N. and Buckleton, J. S. Forensic Interpretation of Glass Evidence. London: CRC Press, 2000.

[10] Petterd, C. I., Hamshere, J., Stewert, S., Brinch, K., Masi, T., and Roux, C. Glass particles found in the clothing of members of the public in south-eastern Australia - a survey. Forensic Science International 1999; 103: 193-198.

[11] Allen, T. J. and Scranage, J. K. The transfer of glass - part 1: transfer of glass to individuals at different distances. Forensic Science International 1998; 93: 167-174.

[12] Allen, T. J., Hoefler, K. and Rose, S. J. The transfer of glass - part 2: the study of the transfer of glass to a person by various methods. Forensic Science International 1998a; 93: 175-193.

[13] Allen, T. J., Hoefler, K. and Rose, S. J. The transfer of glass - part 3: the transfer of glass from a contaminated person to another uncontaminated person during a ride in a car. Forensic Science International 1998b; 93: 195-200.

[14] Allen, T. J., Cox, A. R., Barton, S., Messam, P. and Lambert, J. A. The transfer of glass - part 4: the transfer of glass fragments from the surface of an item to a person carrying it. Forensic Science International1998; 93: 201-208.

[15] Hicks, T., Vanina, R. and Margot, P. Transfer and Persistence of Glass Fragments on Garments. Science and Justice 1996; 36 (2): 101-107.

[16] Brewster F, Thorpe J, Gettinby G, Caddy B The retention of glass particles on woven fabrics. Journal of Forensic Sciences 1985; 30 (3):798-805.

[17] Pearson, G. F., May, R. W. and Dabbs, M. D. G. Glass and Paint Fragments Found in Men's Outer Clothing - Report of a Survey. Journal of Forensic Sciences 1971; 16(3):283-300. 
[18] Wiggins, K. G., Emes, A. and Brackley, L. H. The transfer and persistence of small fragments of polyurethane foam onto clothing. Science and Justice 2002;

42:105-110

[19] Dachs, J., McNaught, L. J. and Robertson, J. The persistence of human scalp hair on clothing fabrics. Forensic Science International 2003; 138:27-36

[20] Whelan, P. M. and Hodgson, M. J. Essential Principles of Physics. Hazell, Watson and Viney, Great Britain, 1989. 